\title{
CXCR4 inhibition in human pancreatic and colorectal cancers induces an integrated immune response
}

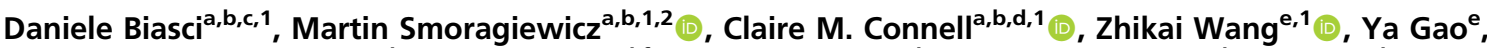 \\ James E. D. Thaventhiran ${ }^{a, b, c} \mathbb{D}_{\text {, Bristi Basu }}^{\text {d,f }}$, Lukasz Magiera ${ }^{a, b, 3}$, T. Isaac Johnson ${ }^{a, b}$, Lisa Bax ${ }^{d}$, Aarthi Gopinathan $^{a, b}$, \\ Christopher Isherwood $^{a, b}$, Ferdia A. Gallagher ${ }^{\text {(D) }}$, Maria Pawula ${ }^{a, b}$, Irena Hudecova $a^{a, b}$, Davina Gale ${ }^{a, b}$, \\ Nitzan Rosenfeld ${ }^{\mathrm{a}, \mathrm{b}}$, Petros Barmpounakis ${ }^{\mathrm{h}}{ }$, Elizabeta Cristina Popa', Rebecca Brais ${ }^{\mathrm{j}}$, Edmund Godfrey ${ }^{\mathrm{g}}$, Fraz Mir $^{\mathrm{k}}$, \\ Frances M. Richards ${ }^{a, b}{ }_{(\mathbb{D}}$, Douglas T. Fearon ${ }^{a, e, i, 4,5}$, Tobias Janowitz $^{a, b, e, l, 4,5} \mathbb{D}_{(\mathbb{D})}$, and Duncan I. Jodrell ${ }^{a, b, 4}$ \\ ${ }^{a}$ Cancer Research UK Cambridge Institute, University of Cambridge, Cambridge, CB2 ORE, UK; ${ }^{b}$ Cancer Research UK Centre-Cambridge, Cancer Research \\ UK Cambridge Institute, Cambridge CB2 ORE, UK; 'Medical Research Council Toxicology Unit, University of Cambridge, Cambridge, CB2 1QW, UK; \\ ${ }^{\mathrm{d}}$ Department of Oncology, Cambridge University Hospitals NHS Foundation Trust, CB2 0QQ Cambridge, UK; ${ }^{\circ}$ Cold Spring Harbor Laboratory, Cold Spring \\ Harbor, NY 11724; fDepartment of Oncology, University of Cambridge, Cambridge Biomedical Campus, Cambridge, CB2 0XZ, UK; ${ }^{9}$ Department of \\ Radiology, Cambridge University Hospitals NHS Foundation Trust, CB2 OQQ Cambridge, UK; ${ }^{\mathrm{h}}$ Department of Statistics, Athens University of Economics and \\ Business, 10434 Athens, Greece; 'Weill Cornell Medicine, New York, NY 10065; 'Department of Pathology, Cambridge University Hospitals NHS Foundation

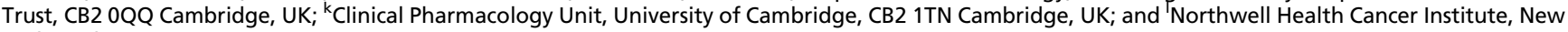 \\ Hyde Park, NY 11042
}

Contributed by Douglas T. Fearon, September 14, 2020 (sent for review July 10, 2020; reviewed by Stephen P. Schoenberger and Charles Swanton)

Inhibition of the chemokine receptor CXCR4 in combination with blockade of the PD-1/PD-L1 T cell checkpoint induces T cell infiltration and anticancer responses in murine and human pancreatic cancer. Here we elucidate the mechanism by which CXCR4 inhibition affects the tumor immune microenvironment. In human immune cell-based chemotaxis assays, we find that $\mathrm{CXCL} 12$ stimulated CXCR4 inhibits the directed migration mediated by CXCR1, CXCR3, CXCR5, CXCR6, and CCR2, respectively, chemokine receptors expressed by all of the immune cell types that participate in an integrated immune response. Inhibiting CXCR4 in an experimental cancer medicine study by $1-w k$ continuous infusion of the small-molecule inhibitor AMD3100 (plerixafor) induces an integrated immune response that is detected by transcriptional analysis of paired biopsies of metastases from patients with microsatellite stable colorectal and pancreatic cancer. This integrated immune response occurs in three other examples of immunemediated damage to noninfected tissues: Rejecting renal allografts, melanomas clinically responding to anti-PD1 antibody therapy, and microsatellite instable colorectal cancers. Thus, signaling by CXCR4 causes immune suppression in human pancreatic ductal adenocarcinoma and colorectal cancer by impairing the function of the chemokine receptors that mediate the intratumoral accumulation of immune cells.

pancreatic cancer | colorectal cancer | CXCR4 | immunotherapy | AMD3100

T cell checkpoint antagonists that target the regulatory membrane proteins on T cells, CTLA-4 and PD-1, have demonstrated the therapeutic potential of the immune system in cancer. Clinical responses, however, have been limited to subsets of patients with certain cancers (1-4). Lack of cancer cell antigenicity (5), dysfunction of cytotoxic $\mathrm{CD}^{+} \mathrm{T}$ cells (6), and systemic immune modulation $(7,8)$ have been some of the potential explanations for resistance of these cancers to $\mathrm{T}$ cell checkpoint inhibitors. A more general immunological principle in which mesenchymal cells may control the immune response to immunogenic epithelial tissues should also be considered (9).

The presence of tertiary lymphoid structures (TLSs) in human adenocarcinomas correlates with better long-term clinical outcome and clinical response to T cell-checkpoint inhibitors (10-13), suggesting that organized intratumoral lymphoid structures promote antitumor immune reactions. Mesenchymal stromal cells organize $\mathrm{B}$ and $\mathrm{T}$ cells in both secondary and TLSs mainly by producing chemokines: CCL19 and CCL21 from fibroblastic reticular cells (FRCs) recruit CCR7-expressing lymphocytes and dendritic cells (DCs), and CXCL13 from follicular DCs (FDCs) attracts CXCR5-expressing T and B cells (14). Notably, these two stromal cell types develop from an embryonic precursor that expresses the membrane protein, fibroblast activation protein- $\alpha$ (FAP), and may be developmentally related to the FAP-expressing

\section{Significance}

Patients with microsatellite stable (MSS) pancreatic (PDA) or colorectal cancer (CRC) do not respond to immunotherapy with inhibitors of $\mathrm{T}$ cell checkpoints. A possible explanation is suggested by finding that cancer cells in these tumors are coated with the chemokine, CXCL12, and that stimulation of CXCR4, the CXCL12 receptor on immune cells, suppresses directed migration mediated by other chemokine receptors on these cells. We assessed the relevance of these findings by treating patients for seven days with continuous infusion of AMD3100/Plerixafor, a CXCR4 inhibitor. Comparison of pre- and end-of-treatment paired biopsies of metastatic lesions by transcriptomic analysis revealed that AMD3100 induced an integrated immune response that is predictive of a clinical response to $T$ cell checkpoint inhibition.

Author contributions: D.B., M.S., C.M.C., Z.W., J.E.D.T., B.B., D.T.F., T.J., and D.I.J. designed research; D.B, M.S, CM.C, ZW Y.G, B.B, L. T.IJ, B, A.G, CI, F.A.G, M.P., I.H D.G., N.R., E.C.P., R.B., E.G., F.M., F.M.R., T.J., and D.I.J. performed research; D.B., M.S., C.M.C., Z.W., Y.G., J.E.D.T., B.B., L.M., T.I.J., F.A.G., M.P., I.H., D.G., N.R., P.B., R.B., E.G., F.M., F.M.R., D.T.F., T.J., and D.I.J. analyzed data; C.M.C. and E.C.P. performed clinical trial delivery; L.B. and A.G. provided clinical trial coordination; B.B. and D.I.J. provided clinical trial supervision; and D.B., D.T.F., T.J., and D.I.J. wrote the paper.

Reviewers: S.P.S., La Jolla Institute for Allergy and Immunology; and C.S., Cancer Research UK.

Competing interest statement: Sanofi provided study drug for the clinical trial and validation of the pharmacokinetics assay, but had no part in study design, data acquisition, data analysis, or manuscript preparation. N.R. and D.G. are co-founders, shareholders, and officers or consultants of Inivata Ltd., a cancer genomics company that commercializes ctDNA analysis. Inivata had no role in the conceptualization, study design, data collection and analysis, decision to publish, or preparation of the manuscript.

This open access article is distributed under Creative Commons Attribution-NonCommercialNoDerivatives License 4.0 (CC BY-NC-ND).

'D.B., M.S., C.M.C., and Z.W. contributed equally to this work.

${ }^{2}$ Present address: Odette Cancer Center, Sunnybrook Health Sciences Centre, Toronto, ON OM N4M, Canada.

${ }^{3}$ Present address: Research and Development Oncology, AstraZeneca, CB2 0QH Cambridge, UK.

${ }^{4}$ D.T.F., T.J., and D.I.J. contributed equally to this work.

${ }^{5}$ To whom correspondence may be addressed. Email: dfearon@cshl.edu or janowitz@ cshl.edu.

This article contains supporting information online at https://www.pnas.org/lookup/suppl/ doi:10.1073/pnas.2013644117/-/DCSupplemental. 
fibroblastic stromal cell type that resides in solid tumors, which is termed the cancer-associated fibroblast (CAF) (15-18). CAFs also affect the trafficking of lymphocytes by producing a chemokine, CXCL12 (19), but in a manner that opposes the effects of lymphoid tissue stromal cells by suppressing the intratumoral accumulation of $\mathrm{T}$ cells (20). Indeed, continuous inhibition of CXCR4 in a mouse model of pancreatic cancer leads to $\mathrm{T}$ cell infiltration of the tumors and results in response to anti-PD-L1 antibody administration (20). Therefore, whether the tumor stroma supports or suppresses immune activation may depend on the relative contributions of these related stromal cell types. A predominance of CAFs would suppress local immunity, whereas the presence of FRCs and FDCs and the development of TLSs would enhance intratumoral immunity. This concept has been supported by preclinical studies in which immune control of tumor growth in mice occurred after $\mathrm{FAP}^{+} \mathrm{CAFs}$ were conditionally depleted $(18,20)$.

Here we report the results from a proof-of-concept experimental medicine study in which we tested the immunological consequences of inhibiting CXCR4 in patients with cancers that have historically resisted immunotherapy, microsatellite stable (MSS) colorectal cancer (CRC) or pancreatic ductal adenocarcinoma (PDA). We report that continuous administration for 1 wk of AMD3100 (plerixafor, Mozobil), a small-molecule inhibitor of $\mathrm{CXCR} 4$, promotes an integrated immune response (INTIRE) in metastatic lesions from these patients.

\section{Results}

Colorectal and Pancreatic Cancer Cells Display a CXCL12 Coat. In murine PDA tumors, cancer cells display a "coat" of CXCL12, the chemokine that $\mathrm{FAP}^{+} \mathrm{CAFs}$ produce to mediate their immune suppressive activity (20). We assessed whether such a CXCL12-coat is displayed on human PDA or CRC cancer cells by examining tumor tissue microarrays. Fluorescently labeled anti-CXCL12 antibodies stained the KRT19-expressing cancer cells in formalin fixed paraffin-embedded (FFPE) tissue sections of human PDA and CRC (Fig. 1).

CXCL12-Stimulated CXCR4 Inhibits the Directed Migration of Human Immune Cells Mediated by Chemokine Receptors. We assessed whether CXCL12 stimulation of CXCR4 altered the trafficking of human immune cells by performing in vitro transwell migration assays using Boyden chambers. We generated human cell lines that coexpressed CXCR4 with the chemokine receptors that mediate the directed migration of innate and adaptive immune cells: CXCR1 in Jurkat-CXCR4/CXCR1 cells, CXCR3 in
HSB2DP-CXCR4/CXCR3 cells, CXCR5 in Raji-CXCR4/ CXCR5 cells, CXCR6 in Jurkat-CXCR4/CXCR6 cells, and CCR2 in Molm13-CXCR4/CCR2 cells, respectively (Fig. 2 and SI Appendix, Fig. S1). These chemokine receptors and their respective chemokines mediate the directed migration of: Neutrophils (CXCR1; CXCL8); T cells, DCs, and NK cells (CXCR3; CXCL9 and CXCL10); B cells (CXCR5; CXCL13); tissueresident memory $\mathrm{T}$ cells (CXCR6; CXCL16); and monocytes and macrophages (CCR2; CCL2) (Fig. 2). Including CXCL12 in the upper chamber of the Boyden two-chamber assay inhibited the migration of the human immune cells coexpressing CXCR4 with each one of the five other chemokine receptors toward its relevant chemokine in the lower chamber. The inhibition was dependent on CXCR4 expression (SI Appendix, Fig. S1) and was unidirectional, in that stimulating the relevant immune cell lines with their respective chemokines for CXCR1, CXCR3, CXCR5, CXCR6, or CCR2 did not abolish the CXCR4-mediated chemotactic response to CXCL12 (Fig. 2), with the exception of partial inhibition by CXCL13. Incubating HSB2DP-CXCR4/ CXCR3 cells with CXCL12 followed by removal of the chemokine restored the ability of cells to migrate in response to a CXCL10 gradient (SI Appendix, Fig. S2).

AMD3100 Suppresses CXCL12-Stimulated Inhibition of Other Chemokine Receptors. In previous murine studies, a continuous plasma concentration of $2 \mu \mathrm{g} / \mathrm{mL}(4 \mu \mathrm{M})$ AMD3100 unmasked anti-PDA immunity and led to reduced tumor growth rates and synergy with anti-PD-L1 treatment (20). We thus examined the effect of AMD3100 in chemotaxis studies across this range of drug concentration in these human cell lines. AMD3100 fully inhibited the CXCR4-mediated chemotactic responses of all immune cell lines (SI Appendix, Fig. S3). The CXCR4 inhibitor also fully reversed the inhibition by CXCL12 of the chemotactic functions of CXCR1, CXCR5, and CXCR6 on the CXCR4 expressing Jurkat cells (Fig. 3). The functions of CXCR3 and CCR2 were only partially restored, which correlated with the inhibitory effects of AMD3100 on CXCR3- and CCR2- mediated chemotaxis in the absence of CXCL12 (SI Appendix, Fig. S3). This inhibitory effect of AMD3100 may be caused by partial agonism of CXCR4, which has been reported previously (21). Since CXCR1, CXCR3, CXCR5, CXCR6, and CCR2 mediate the trafficking of neutrophils, T cells, NK cells, DCs, B cells, tissue-resident memory $\mathrm{T}$ cells, and monocytes, these observations suggest that AMD3100 may alter the trafficking of multiple immune cell types within tumors, thereby inducing an INTIRE to
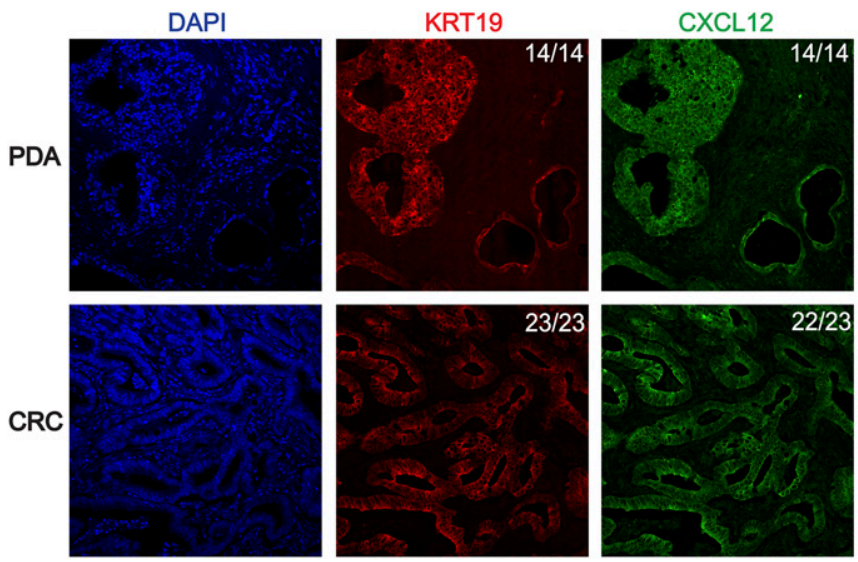
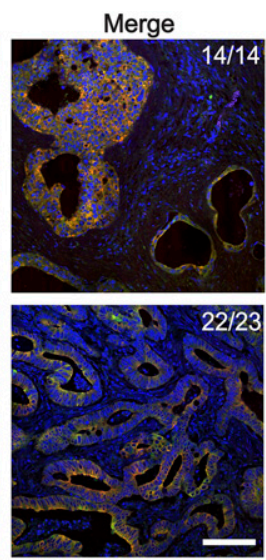

Fig. 1. The CXCL12-coat of human pancreatic and colorectal cancer cells. Sections of human pancreatic (PDA) and colorectal (CRC) adenocarcinoma were stained with fluorescent antibodies to CXCL12, and to KRT19 to reveal cancer cells. The ratios shown in the top right corners of the photomicrographs indicate the frequency of the observed staining relative to the total number of independent tumors that were assessed. (Scale bar, $50 \mu \mathrm{m}$.) 

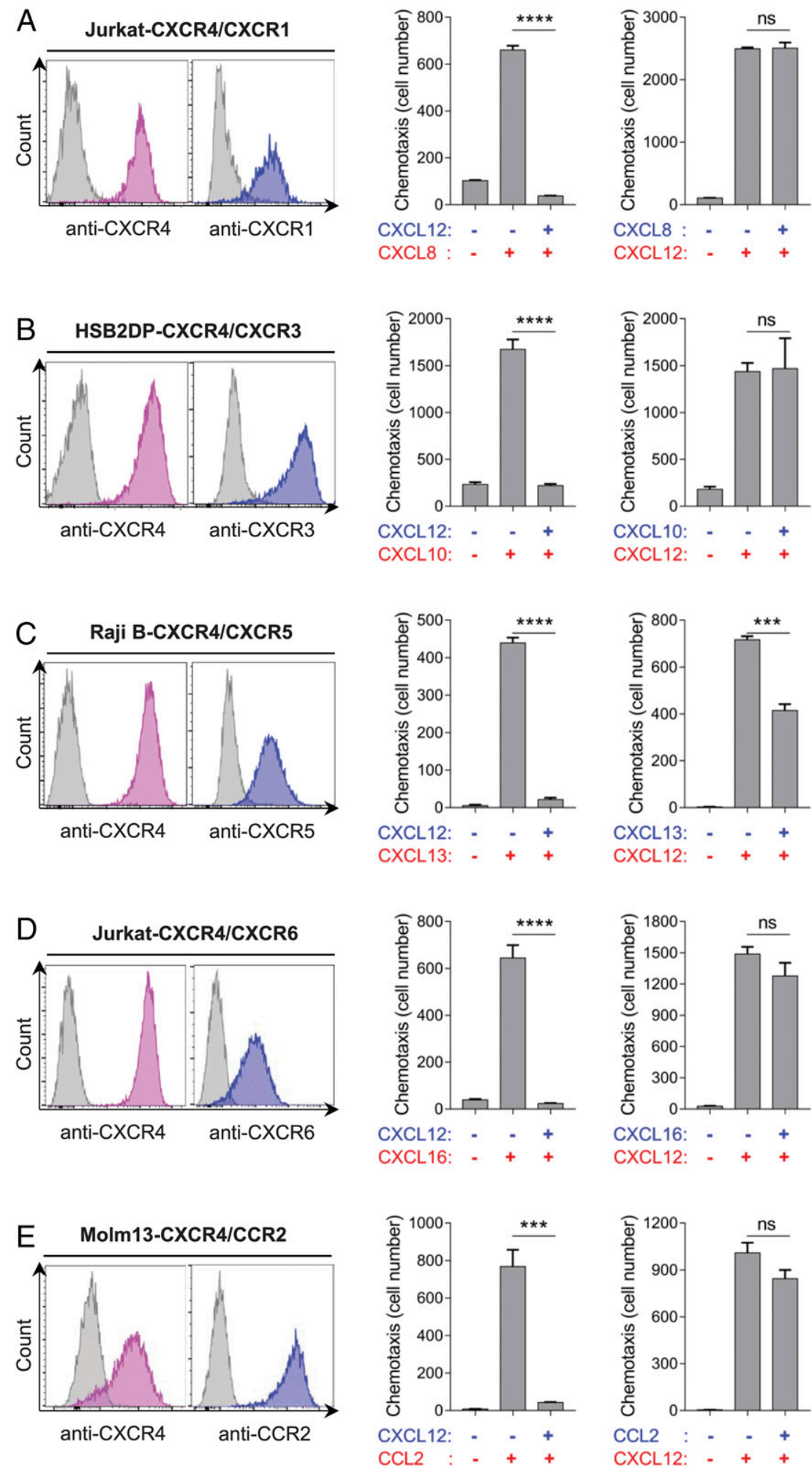

Fig. 2. The effect of CXCL12-stimulated CXCR4 on chemokine receptor-mediated migration of human immune cells. (Left) The coexpression of CXCR4 with $(A)$ CXCR1, (B) CXCR3, (C) CXCR5, (D) CXCR6, and (E) CCR2 on human immune cell lines was evaluated by flow cytometry after staining with antibodies specific for the relevant chemokine receptors. Gray peaks indicate isotype controls. (Center) The effect of CXCL12-stimulation of CXCR4 on the chemotactic responses of $A$ CXCR1-coexpressing Jurkat T lymphoblastoid cells to CXCL8, $(B)$ CXCR3-coexpressing HSB2DP T lymphoblastoid cells to CXCL10, (C) CXCR5-coexpressing Raji B lymphoblastoid cells to CXCL13, $(D)$ CXCR6-coexpressing Jurkat T lymphoblastoid cells to CXCL16, and (E) CCR2-coexpressing Molm13 monocytoid cells to CCL2 was assessed by including CXCL12 in the upper chamber (blue) and the other chemokines in the lower chamber (red) in the Boyden two-chamber assay. (Right) The chemotaxis assays were performed with the five cell lines when the placement of the chemokines in the Boyden chambers was reversed. Bar diagrams display mean and SEM $(\mathrm{n}=3-4)$. Statistical analysis by Student's $t$ test: $* \star * P<0.001 ; * \star * * P<0.0001$; ns, not significant. 

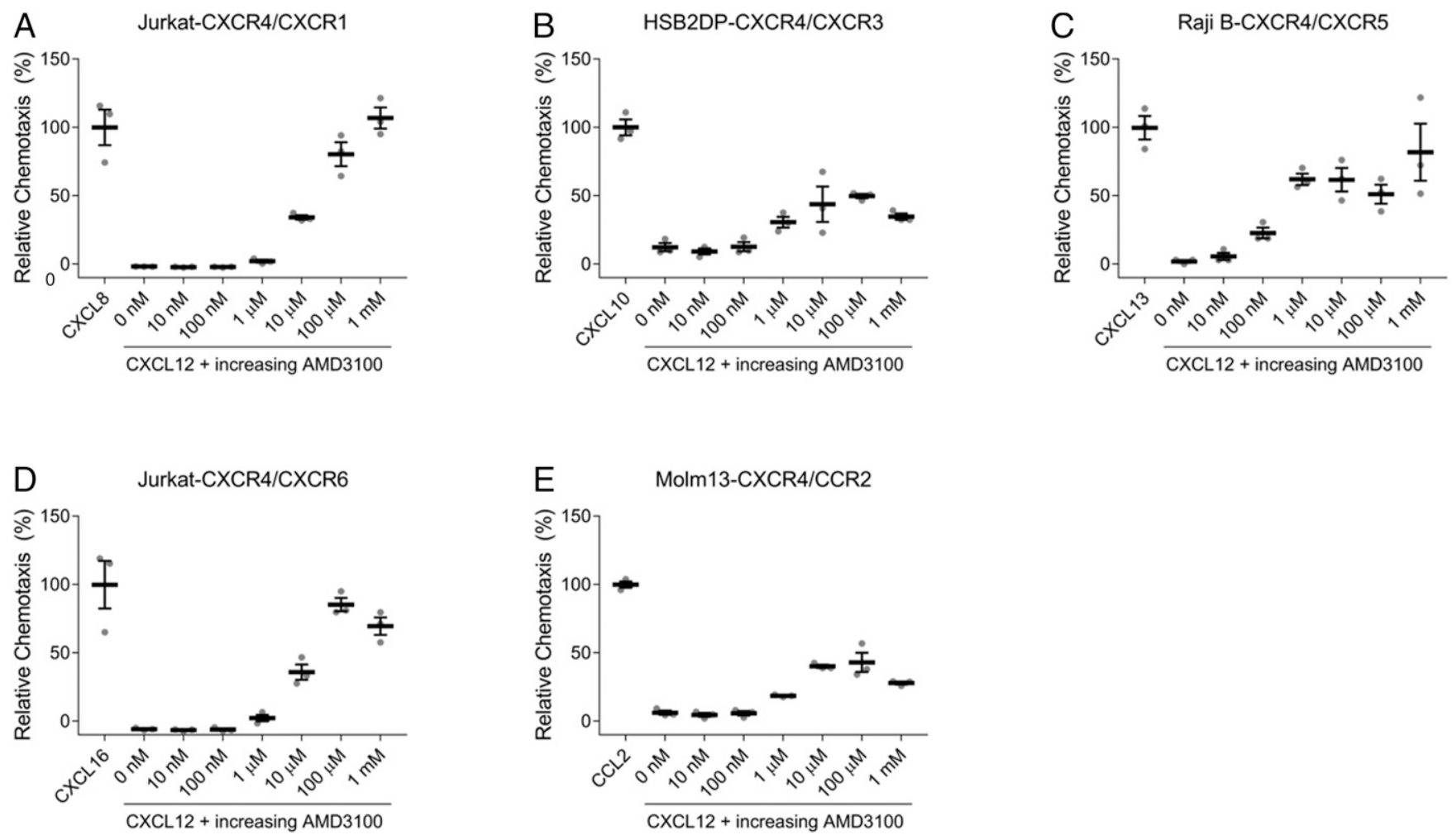

Fig. 3. Inhibition by AMD3100 of the suppression mediated by CXCL12-stimulated CXCR4 of the function of other chemokine receptors. ( $A-E$ ) The chemotactic responses were assessed of the dual chemokine receptor-expressing human immune cells to the relevant chemokines in the absence or presence of CXCL12, with increasing concentrations of AMD3100. The results are presented as percent of the chemotactic response in the absence of CXCL12 and AMD3100. The mean and SEM are indicated.

the cancer cells. We tested this hypothesis in an experimental medicine study.

Experimental Medicine Study of Continuous AMD3100 Infusion: Study Design, Recruitment, and Patient Characteristics. We targeted the CXCL12/CXCR4 interaction using AMD3100 in an experimental medicine study of the immunological role of CXCR4 signaling in patients with MSS CRC and MSS PDA (NCT02179970). AMD3100 has a plasma half-life of $\sim 8 \mathrm{~h}$. To achieve continuous inhibition of CXCR4, as has been recommended for other chemokine receptors (22), AMD3100 was delivered by continuous intravenous infusion for $7 \mathrm{~d}$ with the target steady-state plasma concentration being $\sim 2 \mu \mathrm{g} / \mathrm{mL}(4 \mu \mathrm{M})$. We assessed the pharmacokinetics, pharmacodynamics, and intratumoral immunological changes during treatment using serial blood tests, clinical imaging modalities, and investigations from paired biopsies taken prior to and at the end of the AMD3100 infusion (SI Appendix, Figs. S4 and S5A).

We enrolled 26 patients at two centers, 24 at the Cambridge University Hospitals National Health Service (NHS) Foundation Trust and two at Weill Cornell Medicine/New York Presbyterian Hospital. The patient eligibility criteria are shown in Methods. The characteristics of all enrolled patients are summarized in Table 1. On histopathological review, one patient was found to have predominantly neuroendocrine cancer cells in the biopsy tissue and this patient was therefore excluded from all analyses other than the safety and pharmacokinetic analysis. The remaining 25 patients had treatment-refractory, histologically confirmed MSS PDA $(n=10)$ or MSS CRC $(n=15)$. An important inclusion criterion was the presence of a baseline lymphocyte count above the lower limit of normal $\left(1.0 \times 10^{9} / \mathrm{L}\right)$ at screening, because of concerns relating to adequate immune status and resolution of immunosuppression after previous chemotherapy. Twenty-four patients with CRC or PDA were treated with AMD3100 (one registered patient did not commence study drug, because of a disease related adverse event [AE]): 17 in the dose escalation phase (2 PDA, 15 CRC) and 7 additional patients with PDA in the dose-expansion phase. We confirmed the presence of the CXCL12-coat in all patients enrolled in the dose-escalation phase who had evaluable tissue (SI Appendix, Fig. S6).

Pharmacokinetic and Toxicity Results. The first dose level of AMD3100 was an intravenous infusion at a rate of $20 \mu \mathrm{g} / \mathrm{kg} / \mathrm{h}$, with subsequent patients enrolled at dose cohorts of 40,80 , and $120 \mu \mathrm{g} / \mathrm{kg} / \mathrm{h}$, using a $3+3$ design. There were no dose-limiting toxicities (DLTs) identified in the $20-, 40-$, and $80-\mu \mathrm{g} / \mathrm{kg} / \mathrm{h}$ dose cohorts, but two patients experienced DLTs at the $120-\mu \mathrm{g} / \mathrm{kg} / \mathrm{h}$ dose (SI Appendix, Table S1). One patient had a vasovagal reaction (grade 3 ) in the context of pain shortly after the day 8 biopsy and prior to completion of the AMD3100 infusion. One patient who had peritoneal disease developed severe abdominal pain (grade 3), hypotension (grade 3 ), and a vasovagal reaction (grade 3 ) on day 2 of the infusion. Symptoms resolved within $24 \mathrm{~h}$ after discontinuing the drug, medications for pain control, and intravenous fluids. Continuous infusion of AMD3100 has been reported to be associated with vasovagal reactions (23), and these events were classified as DLTs. A complete list of graded AEs is included in SI Appendix (SI Appendix, Table S2).

In the trial of continuous intravenous infusion of AMD3100 for $1 \mathrm{wk}$ in 40 patients with HIV, a single patient experienced premature ventricular contractions (23). Thus, in the present study, all patients were admitted for the initial $72 \mathrm{~h}$ of the AMD3100 infusion for continuous cardiac telemetry monitoring, 
Table 1. Patient characteristics

\begin{tabular}{lc} 
Characteristic & $n=26$ \\
\hline Age, y & \\
Mean & 66 \\
Range & $50-76$ \\
Female sex, $n(\%)$ & $9(35)$ \\
Histology, $n(\%)$ & \\
Pancreatic adenocarcinoma & $10(38)$ \\
Colorectal adenocarcinoma & $15(58)$ \\
Neuroendocrine cancer* & $1(4)$ \\
ECOG, $n(\%)$ & \\
0 & $9(35)$ \\
1 & $17(65)$ \\
Lymphocyte count (10\%) & \\
Median & 1.41 \\
Range & \\
Albumin $<35$ g/L, $n(\%)$ & $0.82-2.31$ \\
Sites of metastasis $>2, n(\%)$ & $13(50)$ \\
Prior lines of chemotherapy, $n(\%)$ & $16(62)$ \\
0 & \\
1 & $1(4)$ \\
2 & $1(4)$ \\
$\geq 3$ & $16(62)$ \\
\hline
\end{tabular}

* On central review of research biopsies, pathology consistent with neuroendocrine pancreatic cancer, excluded from later analysis.

${ }^{\dagger}$ One patient had a low count at enrollment that normalized on day 1 preinfusion.

and Holter monitoring thereafter. No cardiac rhythm disturbances were identified at the $80-\mu \mathrm{g} / \mathrm{kg} / \mathrm{h}$ infusion rate chosen for the expansion phase. Minor changes (SI Appendix, Table S2) were only possibly drug related, and there were no cardiac AEs that required drug interruption and all resolved without sequelae. Therefore, hospital-based telemetry is not indicated in future studies using this infusion protocol and static ECGs and ambulatory Holter monitoring should provide sufficient cardiac monitoring.

The dose rate of $80 \mu \mathrm{g} / \mathrm{kg} / \mathrm{h}$ yielded the target plasma level of $\sim 2 \mu \mathrm{g} / \mathrm{mL}(4 \mu \mathrm{M})$, and was chosen for the expansion cohort, which resulted in a mean steady-state plasma concentration of $2.3 \mu \mathrm{g} / \mathrm{mL}(\mathrm{SD} \pm 0.9 \mu \mathrm{g} / \mathrm{mL})$ AMD3100 in patients enrolled at this dose rate (SI Appendix, Fig. S5B and Table S3). This infusion rate was overall well tolerated (SI Appendix, Table S2).

Pharmacodynamic and Clinical Results. In accordance with the wellcharacterized biological role of CXCL12/CXCR4 ligation for the retention of hematopoietic stem cells (HSCs) and immature leukocytes in the bone marrow (24), CD34 ${ }^{+}$and other leukocyte populations were elevated during the period of AMD3100 infusion. These changes had almost completely resolved by day 28 of the study, $20 \mathrm{~d}$ after discontinuation of the infusion ( $\mathrm{SI} \mathrm{Ap}$ pendix, Fig. S7). By conventional computed tomography (CT) scanning on days 20 to 24 , no complete or partial responses by RECIST 1.1 were identified in 23 evaluable patients. Thirteen patients $(57 \%)$ had stable disease and $10(43 \%)$ disease progression. Paired positron emission tomography (PET-CT) scans were evaluable in 19 participants (12 escalation phase, 7 expansion phase). Of these, 11 participants had CRC and 8 PDA. Clinically significant (defined as delta standardized uptake values mean weighted average [SUV MWA] $\geq 30 \%$ ) changes were seen in two participants. Both were patients with CRC (treated at $40 \mu \mathrm{g} / \mathrm{kg} / \mathrm{h})$ and had a $\geq 30 \%$ increase in SUV MWA $(71 \%$ and $32 \%$ ).

Immunological Analysis of Metastatic Tissue from Pretreatment and On-Treatment Biopsies. We performed immunological analyses on all patients who had sufficient sample material for comparative histopathological and RNA studies (PDA $n=4$; CRC $n=10)(S I$ Appendix, Fig. S4). We first sought histological evidence of an immunological response to CXCR4 inhibition by determining the frequency of $\mathrm{CD}^{+} \mathrm{T}$ cells in paired pre- and end-of-treatment biopsies of metastatic lesions. FFPE tissue sections were stained with antibodies specific for CD8 $\alpha$ and keratin (pan-CK), respectively (Fig. 4A). There was a significant increase in the number $\mathrm{CD}^{+} \mathrm{T}$ cells in the pan- $\mathrm{CK}^{+}$cancer cell areas after treatment with AMD3100 (SI Appendix, Fig. S8A). Frozen samples of different biopsy passes of the same metastatic lesions were subjected to bulk RNA-sequencing (RNA-seq) analysis. The CD8a mRNA levels were significantly up-regulated after AMD3100 administration (SI Appendix, Fig. S8B), and significantly correlated with the histopathologically determined $\mathrm{CD}^{+} \mathrm{T}$ cell frequencies (Fig. 4B). This validation of the quantitative RNA-seq transcriptomic set justified the further analysis of this comprehensive source of immunological data.

By enrichment analysis of the RNA-seq data, we found that continuous inhibition of CXCR4 by infusion of AMD3100 induced intratumoral $\mathrm{T}$ and NK cell accumulation and activation (Fig. 4C) and also induced an activated B cell response. Eighteen of the 100 most differentially expressed genes were derived from the $\mathrm{B}$ cell lineage. The up-regulated expression of transcripts encoding the $\mathrm{J}$ chain, heavy-chain constant regions, and TNFRSF17, when taken together with the more modest increased expression of MS4A1 (CD20), CD19, TNFRSF13B, and TNFRSF13C, suggests that this response is more a consequence of plasma cell differentiation than an accumulation of B cells (Dataset S1). A plasma cell transcriptional signature in breast and lung adenocarcinomas has been shown to correlate with improved survival (25). This AMD3100-induced T and B cell response was accompanied by transcriptional evidence for the development of TLSs (Fig. $4 D$ and $E$ and Dataset S1). The restriction of CCL19 mRNA, which is expressed only by FRCs (26), to tumor stromal cells that were $\mathrm{FAP}^{+}$, a marker not only of CAFs but also of FRCs $(15,27)$, was demonstrated by fluorescent in situ hybridization (FISH). The $\mathrm{FAP}^{+}$cells expressing CCL19 increased significantly from 5.8 to $25.7 \%$ (Fig. $4 D$ ), suggesting either that CAFs were differentiating to FRCs within the tumor microenvironment, or that FRCs were being recruited from a source outside the tumors.

The INTIRE and Immune-Mediated Damage to Noninfected Tissues. The similarity of the AMD3100-induced transcriptional changes to those that characterize tumors with TLSs (Fig. 4E), microsatellite instable (MSI) CRC (Fig. $4 F$ ), and rejecting renal allografts (Fig. 4G) suggested that the inhibition of a single chemokine receptor, CXCR4, may induce an integrated immune reaction that is characteristic of noninfected, immunogenic tissues. To assess the INTIRE, we developed based on prior knowledge from the literature the INTIRE gene signature, which was defined by 194 genes that together identify nine components of innate and adaptive immunity (Dataset S2): Monocyte/macrophage/DC/antigen presentation, $\mathrm{T}$ and NK cell accumulation, $\mathrm{T}$ and NK effector cells, chemokines and chemokine receptors, activated B cells (germinal center B cells) and plasma cells, stromal/FRC/TLS, type I/III IFN response, and endothelial cells (blood and lymphatic). AMD3100 treatment of MSS PDA and MSS CRC patients induced the INTIRE gene signature (Fig. 5A). Up-regulation of the INTIRE gene panel also was associated with decreased expression of E2F target genes and other genes involved in the G2M checkpoint, possibly indicating a reduction in replicating cancer cells in the tumor. The INTIRE gene signature also distinguished between rejecting and nonrejecting renal allografts in two studies $(28,29)$, a prototypical example of immunologically mediated damage to noninfected tissue. Relative to MSS CRC, MSI CRC demonstrated the INTIRE gene signature (Fig. $5 A)(30,31)$, but, in contrast to the 

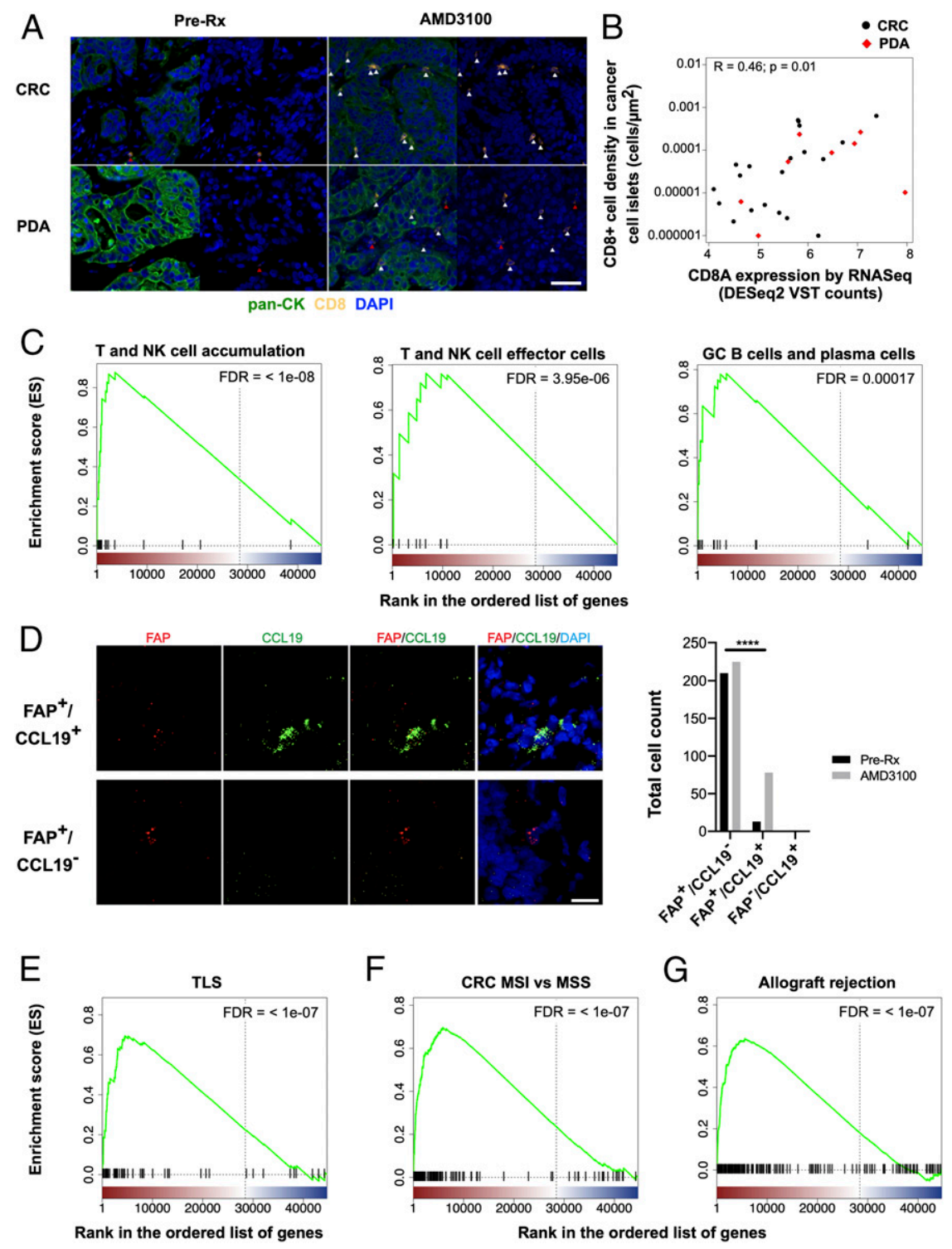

Fig. 4. The immunological effects in human CRC and PDA of treatment with AMD3100. Paired biopsy tissues were obtained from the same metastasis in each patient before (pre-Rx) and after $7 \mathrm{~d}$ of continuous infusion of AMD3100. (A) Tissue sections were stained with fluorescent antibodies to pan-keratin (pan-CK) to reveal cancer cells, and to CD8 to reveal cytotoxic T cells. White arrowheads designate CD8 ${ }^{+} \mathrm{T}$ cells within cancer cell islets, and red arrowheads designate $\mathrm{CD}^{+} \mathrm{T}$ cells outside of cancer cell islets. (Scale bar, $50 \mu \mathrm{m}$.) $(B)$ The presence of $\mathrm{CD} 8^{+} \mathrm{T}$ cells within cancer cell islets, assessed by staining with anti-CD8 antibody, correlates with the CD8A mRNA levels, assessed by RNA-seq analysis, in tissues obtained from different pass biopsies of the same metastatic lesions. (C) Immunological gene sets that identify T and NK cell accumulation, T and NK cell effector cells, activated B cells (germinal center B cells) and plasma cells are enriched in genes up-regulated after treatment with AMD3100. (D) The expression of CCL19 and FAP in sections from paired biopsies was analyzed by FISH using specific probes for mRNA of FAP and CCL19. The total counts of FAP ${ }^{+} / \mathrm{CCL} 19^{+}$cells, $\mathrm{FAP}^{+} / \mathrm{CCL}^{-} 9^{-}$cells, and FAP ${ }^{-} / \mathrm{CCL} 19^{+}$cells are displayed. (Scale bar, 50 $\mu \mathrm{m}$.) (E) The enrichment analysis for a TLS gene set (13) is shown. ( $F$ and $G)$ Enrichment analyses are shown for those genes that $(F)$ are differentially expressed in rejecting compared to nonrejecting kidney allografts $(28,29)$ and $(G)$ MSI compared to MSS CRC $(30)$. $(A-G) n=14$ comprising of PDA $(n=4)$ and CRC $(n=$ 10) Statistical comparisons by Spearman's rank correlation test $(B)$, and by Fisher's exact test $(D): * * * * P<0.0001$.

effects of inhibiting CXCR4 in MSS CRC and MSS PDA, MSI CRC did not exhibit decreased cell cycle gene expression (Fig. 5A). The INTIRE gene signature was also present in tumors from longer surviving patients in the data from the Prediction of Clinical Outcomes from Genomic Profiles (PRECOG) study (Fig. 5B) (25). In two studies of melanoma patients treated with anti-PD-1 antibody after $28 \mathrm{~d}$ and $11 \mathrm{~d}$, respectively (32, 33 ), and a study of melanoma patients treated with combination anti-PD-1 plus anti-CTLA-4 antibodies (33), responders demonstrated the INTIRE gene signature. Remarkably, the INTIRE gene signature also distinguished between melanomas that subsequently responded to treatment with anti-PD-1 antibody from those that did not $(32,33)$ (Fig. $5 A$ and $C$ ). Finally, melanomas in patients who were depleted of B cells by administration of anti-CD20 (34) demonstrated an attenuated INTIRE gene signature (Fig. $5 A$ and $D$ ), exemplifying the integrated nature of this immune response.

Immune-Mediated Anticancer Effects of AMD3100 Administration. We examined the transcriptional changes in the paired biopsies for evidence of intratumoral immune-mediated anticancer effects. Changes in the mRNA levels in biopsies from each patient of 

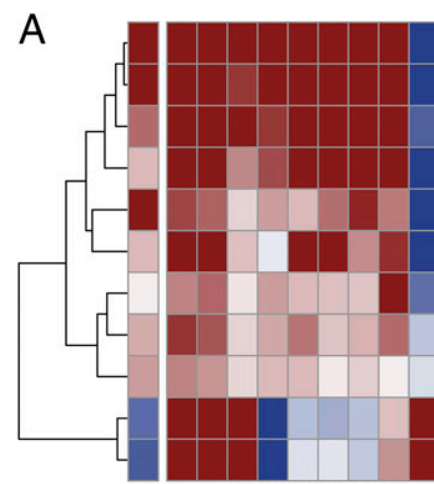

Monocyte / Macrophage / DC / Ag presentation

$\mathrm{T}$ and NK cell accumulation

$T$ and NK cell effector cells

Chemokines and chemokine receptors

Activated B cells (germinal center B cells) and plasma cells

Type I / III IFN Response

$B$ cell accumulation

Stromal / FRC / Tertiary lymphoid structure

Endothelial cells

Cell cycle: E2F targets

Cell cycle: G2M checkpoint

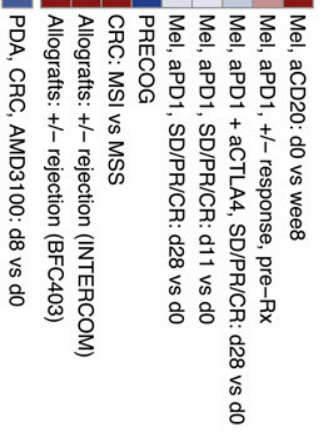

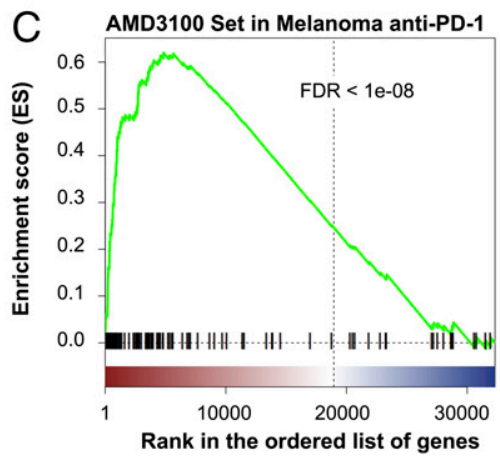

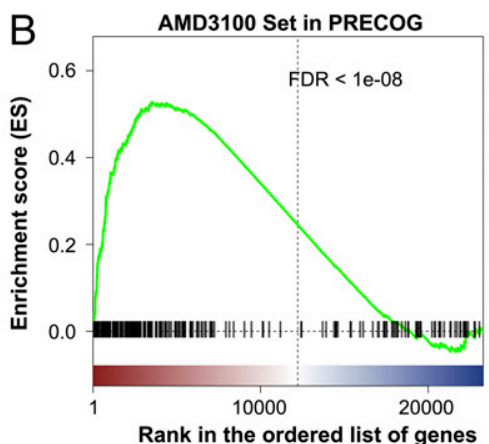

Rank in the ordered list of genes

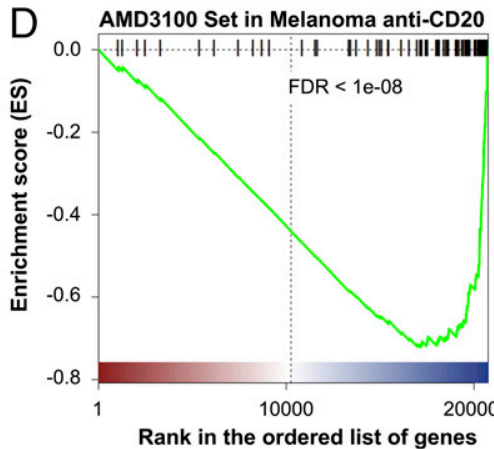

Fig. 5. Comparative analyses of the INTIRE induced by AMD3100. ( $A$ ) The heat map is shown of the enrichment analyses of nine gene sets representing different immune components that characterize the INTIRE in different immunological contexts, along with E2F target genes and genes involved in the G2M checkpoint. Gene set enrichment analyses demonstrating that genes up-regulated by treatment with AMD3100 are significantly enriched in $B$ genes associated with longer overall survival in cancer according to the PRECOG (25) study, $(C)$ genes up-regulated in pretreatment biopsies from patients with melanoma who responded to anti-PD-1 treatment vs. nonresponding patients $(32,33)$, and $(D)$ genes down-regulated in biopsies of patients with melanoma treated with anti-CD20 antibodies to induce B cell depletion (34). (A-D) $n=14$ comprising of PDA $(n=4)$ and CRC $(n=10)$.

granzymes (GZM) A, B, H, K, and M, and perforin, which encode the proteins that mediate killing by effector $\mathrm{CD}^{+} \mathrm{T}$ cells, significantly inversely correlated with changes in the mRNA levels of three genes uniquely expressed by cancer cells, CEACAM 5, 6, and 7 (Fig. 6A), but not with noncancer-specific genes ( $S I A p$ pendix, Fig. S9).

Next, we evaluated plasma biomarker evidence of anticancer effects in all samples that passed the respective quality thresholds for analysis. The plasma concentrations of the tumor-derived markers, carcinoembryonic antigen (CEA) and carbohydrate antigen 19-9 (CA 19-9), which are not validated as early-response markers in immunotherapy trials, were not significantly changed over the 7-d treatment period of the patients with AMD3100 $(n=$ $15, P=0.4)$. Next, we quantified circulating tumor DNA (ctDNA). Levels of ctDNA have been shown to decrease when patients respond to therapy (35-38). We evaluated ctDNA levels at baseline and after $7 \mathrm{~d}$ of treatment with AMD3100 (Fig. 6B). ctDNA levels were significantly reduced following treatment with AMD3100 $(n=15, P=0.033)$. Furthermore, plasma levels of CXCL8, which has also been identified as a marker of tumor
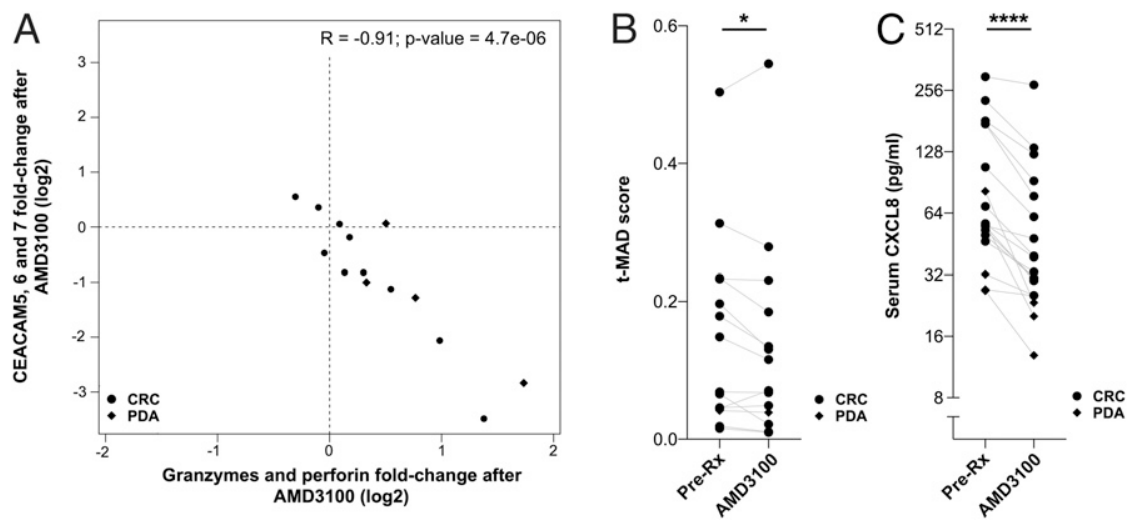

Fig. 6. Analyses of the anticancer effect induced by AMD3100 treatment. (A) Changes of mRNA expression in the paired biopsies obtained from each patient with CRC $(n=10)$ and with PDA $(n=4)$ before and after treatment with AMD3100 for granzymes A, B, H, K, and M and perforin negatively correlate with changes in the expression of CEACAM 5, 6, and 7. (B) Plasma ctDNA levels $(n=15)$ and $(C)$ serum concentrations of CXCL8 $(n=18)$ pretreatment (pre-Rx) and after $7 \mathrm{~d}$ of continuous infusion of AMD3100 are shown. Statistical comparisons by Spearman's rank correlation test $(A)$, by paired Wilcoxon signed-rank test $(B)$, and by paired $t$ test $(C): * P<0.05 ; * * * P<0.0001$. 
burden (39) and may provide an early indicator of therapeutic response (39-41), were also significantly decreased following treatment with AMD3100 (Fig. $6 C)(n=18, P<0.0001)$. The decrease in both ctDNA and CXCL8 levels support the possibility of an early anticancer effect mediated by CXCR4 inhibition.

\section{Discussion}

The findings that the CAF mediates intratumoral immune suppression $(18,20)$ and that a CAF-derived chemokine, CXCL12, coats the cancer cells in PDA and CRC suggest that its receptor, CXCR4, has a role in mediating immune suppression in the tumor microenvironment. The association of CXCL12 with cancer cells is predicted to have two immunological consequences. First, most immune cells in PDA and CRC tumors express CXCR4 and will, therefore, be stimulated by cancer cellassociated CXCL12 via ligation of this receptor. Second, CXCL12-stimulated CXCR4 inhibits the chemotactic functions of the chemokine receptors that direct the migration of immune cells. Therefore, the CXCL12-coat of cancer cells could impair the intratumoral accumulation of multiple immune cell types. We tested these two predictions in an experimental medicine study in which patients with PDA and CRC received the smallmolecule CXCR4 antagonist, AMD3100, which is licensed for mobilization of HSCs.

Treatment of patients with AMD3100 was limited to continuous intravenous infusion of the drug for $1 \mathrm{wk}$, using a protocol shown to be safe in patients with HIV (23). We first confirmed that the AMD3100 infusion did achieve continuous CXCR4 inhibition by observing the presence of $\mathrm{CD} 34^{+} \mathrm{HSCs}$ in the peripheral blood of each patient. All patients from the doseescalation phase showed persistent elevations of $\mathrm{CD} 34^{+} \mathrm{HSCs}$, indicating the occurrence of continuous CXCR4 inhibition, and we therefore included patients from the entire cohort in all subsequent analyses. The short duration of drug administration limited our ability to observe whether CXCR4 inhibition induced clinical responses in patients with PDA or CRC, as assessed by standard radiological evaluations $(42,43)$. These assessments did not reveal remissions and the lack of change in tumor volume is not informative due to the short time period that elapsed between scans. An independent clinical trial testing discontinuous CXCR4 inhibition by subcutaneous administration of a cyclic peptide inhibitor of CXCR4 together with anti-PD-1 antibody over several cycles in patients with advanced pancreatic cancer showed some evidence of clinical responses (44). We observed significant decreases in the levels of ctDNA and circulating CXCL8 of patients after treatment with AMD3100. ctDNA and CXCL8 are increasingly recognized as markers of tumor burden $(35,38,39)$ and may provide early indications of response to therapy (35-41) when imaging evaluation is not conclusive (42, 43). However, these initial observations will require further prospective validation.

This study focused on the question of whether the CXCL12coating of cancer cells in PDA and CRC signified the existence of a fundamental immune suppressive pathway in two human cancers that have thus far resisted cancer immunotherapy. We chose to detect immunological changes by performing bulk RNA-seq analysis of paired biopsies of metastatic lesions taken from patients before and at the end of the AMD3100 infusion. This analysis provided an unbiased and quantitative means of measuring the AMD3100-induced changes in the complex intratumoral immune environments of these tumors, which could be compared to similar transcriptional analyses of tissues representing other immunological reactions.

This comparative transcriptional analysis revealed unanticipated similarities between the immunological effects of two mechanistically distinct immunotherapies, inhibition of $\mathrm{T}$ cell checkpoints and inhibition of a chemokine receptor, respectively, in cancers that have different developmental origins, adenocarcinomas, and melanomas. Both anti-PD-1 and anti-CTLA-4 antibody therapies, which enhance the activation of T cells, and AMD3100 treatment, which affects the trafficking of immune cells, upregulated the expression of genes that characterize rejecting renal allografts, an example of immune damage to immunogenic, noncancer tissue. Thus, effective cancer immunotherapy engages an immune pathway that mediates damage to noninfected, immunogenic tissue. This pathway involves multiple immune elements, and their participation could be assessed by the INTIRE signature, which characterizes nine components of the immune reaction. This analysis showed that the INTIRE signature was induced not only by CXCR4 inhibition in patients with PDA and $\mathrm{CRC}$, but also by successful treatment of patients with melanoma with anti-PD-1 antibody. The occurrence of the INTIRE signature was even predictive of subsequent clinical responses to anti-PD-1 antibody therapy in patients with melanoma. The additional finding that AMD3100 leads to the increased frequency of $\mathrm{FAP}^{+}$cells expressing CCL19, which is a characteristic of FRCs, is consistent with the concept that shifting the balance from immune suppressive fibroblastic cells to those with immuneenhancing functions improves the outcome of cancer immunotherapy. This observation is reminiscent of the recent reports that the presence of TLSs, which in the mouse requires $\mathrm{FAP}^{+}$ fibroblasts $(15,45)$, correlates with clinical responses to $\mathrm{T}$ cell checkpoint therapy (11-13).

Finally, the study raises the possibility that a substantial proportion of patients with MSS PDA and MSS CRC have on-going anticancer immune responses. A majority of the patients treated with AMD3100 showed enhanced intratumoral immune B and $\mathrm{T}$ cell responses after only $7 \mathrm{~d}$, which would be unusually rapid for a primary immune response. Thus, intratumoral immune suppression rather than immune ignorance may be a major barrier to clinically effective immunotherapy. This possibility should be assessed with an appropriate clinical trial of repeat cycles of continuous CXCR4 inhibition in combination with a $\mathrm{T}$ cell checkpoint antagonist.

\section{Methods}

Immunofluorescence of Human Tumor Arrays. FFPE human pancreatic and colorectal tumor arrays (US Biomax) were deparaffinized in xylene, washed three times with ethanol, and rehydrated in a serial concentration of ethanol (from 95 to $50 \%$ ), and finally in water. For antigen retrieval, the sections were boiled in $10 \mathrm{mM}$ Tris, pH 8.8 plus $1 \mathrm{mM}$ EDTA for $10 \mathrm{~min}$, followed by cooling down for $30 \mathrm{~min}$, a wash with PBS, and blocking with $1 \%$ BSA/PBS at room temperature for $1 \mathrm{~h}$. Following two washes with $0.05 \%$ Tween-20/PBS and one with PBS, Alexa Fluor 568-conjugated anti-KRT19 antibody (Abcam, ab203445) and FITC conjugated anti-CXCL12 antibody (R\&D Systems, IC350F) were applied and the sections were incubated at room temperature for $1 \mathrm{~h}$ Finally, the sections were stained with DAPI (Thermo Fisher, R37606) for $10 \mathrm{~min}$ and washed with $0.05 \%$ Tween-20/PBS for two times and once with PBS, followed by application of mounting medium (Thermo Fisher, P36961) and imaging with Leica SP8 confocal microscope. Images were analyzed and exported through ImageJ.

\section{Chemotaxis Assays}

Plasmids. To generate lentiviral plasmid expressing human chemokine receptors, CXCR1 and CXCR3 CDNA from CXCR1-Tango (Addgene, \#66259) and CXCR3-Tango (Addgene, \#66261), respectively, were amplified and subcloned into lentiCas9-blast (Addgene, \#52962) with restriction enzymes Age1 and BamH1 to replace SpCas9; CXCR5 and CXCR6 CDNA from CXCR5-Tango (Addgene, \#66263) and CXCR6-Tango (Addgene, \#66264), respectively, were amplified and subcloned into lentiCas9-puro to replace SpCas9 where blasticidin-resistant gene (blast) was also replaced with puromycin-resistant gene (puro). For CRISPR editing, control guide (sgScramble, GCTTAGTTACGCGTGGACGA) and guides targeting to human CXCR4 gene (sgCXCR4-1, TGACATGGACTGCCTTGCAT; sgCXCR4-2, CAACCACCCACAAGTCATTG; sgCXCR4-3, CAGGACAGGATGACAATACC) or human RGS14 gene (sgRGS14-1, GCAGGGATCTGTGAGAAACG; sgRGS14-2, TCGGCAGCCCTGACGCCACG; sgRGS14-3, CTGAGACTCTCGGCGCAAGG) were cloned into the vector lentiCRISPR-v2 (Addgene, \#62961). 
Cell lines. Jurkat cells (ATCC, Clone E6-1, TIB-152) were transduced with lentivirus expressing human CXCR1, CXCR3, or CXCR6, followed by treatment with $5 \mu \mathrm{g} / \mathrm{mL}$ blasticidin (CXCR1 and CXCR3) or $0.5 \mu \mathrm{g} / \mathrm{mL}$ puromycin (CXCR6) for $2 \mathrm{wk}$, to generate Jurkat-CXCR4/CXCR1 cells, Jurkat-CXCR4/ CXCR3 cells and Jurkat-CXCR4/CXCR6 cells, respectively. For Raji B-CXCR4/ CXCR5 cells, Raji B cells (ATCC, CCL-86) were transduced with CXCR5 lentivirus followed by selection with $0.5 \mu \mathrm{g} / \mathrm{mL}$ puromycin for $2 \mathrm{wk}$. Subpopulation of CCRF-HSB-2 cells (ATCC, CCL 120.1) that spontaneously express a high level of CXCR3 were FACS sorted as HSB2DP-CXCR4/CXCR3 cells, where DP stands for "double positive." The Molm13 cell line (Molm13-CXCR4/CCR2), which expresses both CXCR4 and CCR2 spontaneously, was a gift from C. Vakoc's laboratory, Cold Spring Harbor Laboratory, Cold Spring Harbor, NY. For CRISPR knock-outs, Jukat-CXCR4/CXCR3 and Molm13-CXCR4/CCR2 cells were transduced with lentivirus expressing SpCas9 and control guide (sgScramble), guides targeting to CXCR4 (sgCXCR4) or RGS14 (sgRGS14). HSB2DP-CXCR4/CXCR3 cells were cultured in IMDM medium (ATCC, 30-2005) supplemented with $10 \%$ FBS (Seradigm, 1500-500), 100 units $/ \mathrm{mL}$ penicillin and $100 \mu \mathrm{g} / \mathrm{mL}$ streptomycin. All of the other cell lines were cultured in RPMI-1640 medium (ATCC, 30-2001) plus 10\% FBS and penicillin/streptomycin. Cells were maintained at the density of $1 \times 10^{5}$ to $1 \times 10^{6}$ cells $/ \mathrm{mL}$ Flow cytometry. For each cell line, $1 \times 10^{5}$ to $1 \times 10^{6}$ cells were pellet and washed with cold FACS buffer (PBS, $2 \%$ FBS, and $20 \mathrm{mM}$ Hepes, $\mathrm{pH} 7.4$ ), followed by Fc receptor blocking with Human TruStain FCX (BioLegend, 422301) in the FACS buffer at $4{ }^{\circ} \mathrm{C}$ for $30 \mathrm{~min}$. Cells were then stained with APC-conjugated anti-Human CXCR4 antibody (BioLegend, 306510) and/or Alexa Fluor 488-conjugated anti-Human CXCR1 antibody (BioLegend, 320616), PE-conjugated anti-Human CXCR3 antibody (BioLegend, 353706), FITC-conjugated anti-Human CCR2 antibody (BioLegend, 357215), FITCconjugated anti-Human CXCR5 antibody (BioLegend, 356913), APC conjugated anti-Human CXCR6 antibody (R\&D Systems, FAB699A) for another $30 \mathrm{~min}$ at $4{ }^{\circ} \mathrm{C}$. Then, cells were washed with the FACS buffer twice and analyzed with an BD LSRForsseta cell analyzer.

Chemotaxis assays. The chemotaxis buffer for HSB2DP-CXCR4/CXCR3 cells is IMDM medium plus $0.1 \% \mathrm{BSA}$ and $20 \mathrm{mM}$ Hepes, $\mathrm{pH} 7.5$ and for all other cell lines is RPMI- 1640 medium plus $0.1 \%$ BSA and $20 \mathrm{mM}$ Hepes, pH 7.5. After collection, cells were washed two times and resuspended in the chemotaxis buffer at the density of $1 \times 10^{6} / \mathrm{mL}$, followed by incubation at $37^{\circ} \mathrm{C}$ for $30 \mathrm{~min}$. For the CXCL12 cross-inhibition of the other chemokine-induced chemotaxis, $80 \mu \mathrm{L}$ cells with or without $100 \mathrm{ng} / \mathrm{mL}$ (200 ng/mL for CCR2) recombinant human CXCL12 (R\&D Systems, 350-NS) were loaded in the upper wells of the Boyden chamber plate (VWR, 89089-934; Supplier no. 3388; 5 - $\mu \mathrm{m}$ membrane pore size), and the lower chambers were flowed in $240 \mu \mathrm{L}$ chemotaxis buffer with PBS or the other specific recombinant human chemokine. To test the effect of other specific chemokine on the CXCL12 induced chemotaxis, cells and the specific chemokine were loaded on the upper chamber, with CXCL12 containing chemotaxis buffer loaded in the lower chamber. To evaluate the activity of AMD3100 (Sigma, A5602) on CXCL12 or the other specific chemokine-induced chemotaxis, cells were pretreated with increasing dose of the drug at $37^{\circ} \mathrm{C}$ for $30 \mathrm{~min}$, then the cell/AMD3100 mixture was loaded in the upper chambers, and CXCL12 or other specific chemokine containing chemotaxis buffer was loaded in the lower chambers. For the AMD3100 rescue of the CXCL12 mediated crossinhibition, the AMD3100 pretreated cells together with $100 \mathrm{ng} / \mathrm{mL}$ CXCL12 were loaded in the upper chambers, and chemotaxis buffer containing the other specific chemokine was flowed in the lower chambers. After the experiments were set up, the Boyden chamber plates were incubated at $37{ }^{\circ} \mathrm{C}$ for 2 or $3 \mathrm{~h}$. Then, the number of cells in the lower chamber was counted with a Guava bench-top flow cytometer, for $30 \mathrm{~s}$ at medium flow rate $(\sim 1.2$ $\mu \mathrm{L} / \mathrm{s}$ ). The concentration of each chemokine used is as following: CXCL8 (R\&D Systems, 208-IL), $20 \mathrm{ng} / \mathrm{mL}$; CXCL10 (R\&D Systems, 266-IP), $1,000 \mathrm{ng} / \mathrm{mL}$ CXCL13 (R\&D Systems, 801-CX), 1,000 ng/mL; CXCL16 (R\&D Systems, 976-CX), $50 \mathrm{ng} / \mathrm{mL}$; CCL2 (R\&D Systems, 279-MC), $200 \mathrm{ng} / \mathrm{mL}$

\section{RNA-Seq Analysis.}

Gene-expression quantification and differential gene-expression analysis. Sequences of human transcripts were downloaded from Ensembl release 97 Transcript quantification was performed using Kallisto v0.43 and gene-level count matrices for use in DESeq2 were calculated using tximport as recommended by DESeq2 authors (46). All subsequent analyses on gene expression were performed using $R$ 3.5.0. For differential expression analysis, raw counts were used directly in DESeq2. For other downstream analyses (i.e., fold-change correlation plots) counts data were transformed using the variance stabilizing transformation as implemented in DESeq2.

Gene set enrichment analysis. Gene set enrichment analysis was performed using the fgsea package available in Bioconductor. Gene set enrichment analysis plots were obtained using a modified version of the associated plotEnrichment function. All gene sets used in the manuscript are provided as supplementary tables. Data used for Fig. $5 A$ were obtained from several different sources: The rejecting allografts gene list was obtained from Gene Expression Omnibus (GEO) series GSE48581 (29) and GSE36059 (28); the microsatellite instability gene list was obtained by combining Xena University of California, Santa Cruz (UCSC) TCGA gene expression data (31) and MSI status for the same tumor samples (30); the list of genes associated with pancancer survival was obtained from the PRECOG portal (25); the anti-PD-1 response gene lists were obtained from the GEO: GSE91061 (32) and European Nucleotide Archive: PRJEB23709 (33); the anti-CD20 gene list was obtained from Array Express: E-MTAB-7473 (34).

Code availability. The authors declare that the $\mathrm{R}$ code used to generate the analysis presented in this study is available in Dataset S3.

\section{Experimental Medicine Study.}

Patient eligibility. Patients with advanced or metastatic PDA, high-grade serous ovarian cancer, or CRC, refractory to or declining conventional chemotherapy were eligible for the dose-escalation phase. The 10-patient expansion cohort at the recommended phase 2 dose (RP2D) of $80 \mu \mathrm{g} / \mathrm{kg} / \mathrm{h}$ was restricted to patients with PDA. Other eligibility criteria included a lesion accessible to biopsy, Eastern Cooperative Oncology Group (ECOG) performance status of 0 or 1 , and adequate organ function, including a lymphocyte count above the lower limit of normal. Patients were excluded if they had significant cardiac comorbidities, such as past history of significant rhythm disturbance. Full eligibility criteria can be viewed at https:/clinicaltrials.gov/ct2/show/ NCT02179970. Patients were accrued at Cambridge University Hospitals NHS Foundation Trust and Weill Cornell Medicine/New York Presbyterian Hospital, NY

Study design. This phase 1, multicentre, open-label, nonrandomized study used a $3+3$ dose-escalation design. The primary endpoint was the safety (evaluated using the Common Terminology Criteria for Adverse Events 4.03) of AMD3100 administered, to achieve a plasma AMD3100 concentration at steady state $\geq 2 \mu \mathrm{g} / \mathrm{mL}$ in $\geq 80 \%$ of patients at the RP2D. AMD3100 was administered as a 7-d continuous infusion. DLT was defined as an adverse reaction $(A R) \geq G 3$ occurring within $21 \mathrm{~d}$ of $A M D$ infusion. Secondary endpoints included overall response rate (RECIST 1.1) at $14( \pm 2) \mathrm{d}$ after the infusion, and metabolic changes in tumor using $\left[{ }^{18} \mathrm{~F}\right] \mathrm{FDG}-\mathrm{PET} / \mathrm{CT}$ within $1 \mathrm{~d}$ of infusion completion. Baseline scans were performed within $14 \mathrm{~d}$ before the start of the infusion. Exploratory objectives included the assessment of immune changes in tumor biopsies. Patients were monitored by cardiac telemetry for the initial $48 \mathrm{~h}$ of the infusion (later amended to $72 \mathrm{~h}$ ), followed by Holter monitoring for the remainder of the infusion.

Patients provided written informed consent to Research Ethics Committeeapproved protocol (REC reference 15/EE/0014 at the United Kingdom Center and Institutional Review Board no. 1508016466 at the United States Center), in compliance with Good Clinical Practice, local regulatory requirements, and legal requirements. A clinical trial authorization was obtained from the Medicine and Healthcare Regulatory Authority. The study was sponsored by Cambridge University Hospitals NHS Foundation Trust and the University of Cambridge and Weill Cornell Medicine/New York Presbyterian Hospital. AMD3100 sample and pharmacokinetic analysis. AMD3100 plasma concentration was assessed at the following nominal time points: Predose, 24, 72, and $168 \mathrm{~h}$ of the infusion. A time point at day $7( \pm 2)$ after infusion discontinuation was added from patient 1017 onwards. The concentration data were generated using a liquid chromatography-tandem mass spectrometry (LC-MS/MS) method, that met the requirements of the European Medicines Agency guidance on method validation, performed by the Cancer Research UK Cambridge Institute PK/Bioanalytics Core Facility. A claim of Good Clinical Practice compliance is made for the sample analysis data, pending the demonstration of long-term storage stability, which is on-going at the time of publication. AMD3100 calibration standards were prepared in the range of 40 to $4,000 \mathrm{ng} / \mathrm{mL}$ using blank control human plasma obtained from the NHS blood transfusion service (lower limit of quantification $40 \mathrm{ng} / \mathrm{mL}$ ). After the addition of the internal standard (AMD3100-D4) and EDTA (10 mM), plasma samples, quality control (QC), and calibration standards were extracted by protein precipitation with $1 \%$ formic acid in methanol. A portion of the supernatant was evaporated to dryness and the residue reconstituted in $1 \%$ formic acid in water prior to analysis on the LC-MS/MS. HPLC $\gamma$ was performed with the Shimadzu Nexera $\times 2$ using a Phenomenex Kinetex F5 column $(1.7 \mu \mathrm{m}, 100 \times 2.1 \mathrm{~mm})$ and mobile phases A and B containing $0.1 \%$ formic acid in water or methanol, respectively. MS/MS detection was carried out using a Sciex API6500 mass spectrometer with an electrospray source. QC samples $(120,400$, and $3,000 \mathrm{ng} / \mathrm{mL}$ ) were used to determine the precision (coefficient of variation [\%CV]) and accuracy 
(relative error [\%RE]). QC intraday $\% \mathrm{CV}$ was $\leq 9.8 \%$, and $\% \mathrm{RE}$ ranged between -3.1 to $4.8 \%$. All instrument control and data collection were performed using Analyst v1.6.2, and peak area integration, regression and quantification using MultiQuant v3.0.2. A weighted $(1 / \times 2)$ least-square linear regression was used to construct the calibration line.

Pharmacodynamic Analysis. At baseline, 24/72/168 h of the infusion, and 21 $( \pm 2) d$ after infusion, blood was collected into Sarstedt EDTA blood tubes and $\mathrm{CD} 34^{+}$cells quantified by flow cytometry. The $\mathrm{CD} 34^{+}$absolute count was derived from a bead-based assay using BD Bioscience Trucount tubes and an International Society of Hematotherapy and Graft Engineering gating strategy.

Positron Emission Tomography. PET was performed in conjunction with lowdose CT for attenuation correction and localization purposes. Images were acquired from the midbrain to the knees, $\sim 90(89.7 \pm 21.1) \mathrm{min}$ after the injection of $\sim 370$ (368 \pm 13$) \mathrm{MBq}$ of [18F]fluorodeoxyglucose ([18F]FDG). The baseline [18F]FDG-PET/CT was performed before or at least $24 \mathrm{~h}$ after the core tissue biopsy, and the posttreatment PET-CT was performed within $24 \mathrm{~h}$ after the biopsy on day 8 of the study. Nineteen patients underwent imaging at both timepoints and were evaluated for metabolic changes following treatment. Response was determined by calculating the change in SUV MWA of the [18F]FDG avid target lesions. SUV MWA was calculated as the sum of the product of the SUV mean $75 \%$ and volume for all target lesions, divided by the total target lesion volume. A clinically significant change was defined as change in SUV MWA $\geq 30 \%$.

\section{Biopsy Processing.}

RNA and DNA extraction. Snap-frozen biopsies were embedded in chilled OCT $\left(-4{ }^{\circ} \mathrm{C}\right)$ and allowed to solidify in a cryotome at $-20^{\circ} \mathrm{C}$. Subsequently, 1030 $\mu \mathrm{m}$ sections were cut and collected in RLT buffer for DNA/RNA extraction, followed by six $6-\mu \mathrm{m}$ sections for histologic analysis. The remaining tissue was cut in 30- $\mu \mathrm{m}$ sections until exhausted. All 30- $\mu \mathrm{m}$ sections were extracted for DNA and RNA using the AllPrep DNA/RNA/miRNA Universal Kit (Qiagen, 80224) according to the manufacturer's instructions from tissues, and DNA quantification by fluorometer according to manufacturer instructions (Qubit 3.0, Life Technologies). An H\&E was reviewed by a histopathologist to determine cellular content ( $>40 \%$ cancer cell content).

Histopathologic analyses. For histopathologic analyses, 3- $\mu \mathrm{m}$ FFPE tissue sections were deparaffinized in xylene and rehydrated in an ethanol series. Immunofluorescence staining was performed on the Bond Rx automated platform. Sections were retrieved with Tris EDTA for $20 \mathrm{~min}$ and incubated sequentially with primary and secondary antibody pairs (where applicable) at room temperature for $30 \mathrm{~min}$ or, for CXCL12, $60 \mathrm{~min}$. Slides were counterstained with DAPI and digitalized using the Axio Scan.Z1 (Zeiss) at $20 \times$ ( $0.22 \mu \mathrm{m}$ per pixel). Tumor areas were selected based on review of serial $\mathrm{H \& E}$ sections by a histopathologist: Liver, peritoneal fat, and necrosis were excluded. The HighPlex FL v3.0.1 algorithm in Halo software (v2.3, Indica Labs) was used to automatically detect $\mathrm{CD} 8^{+}$cells across all sections, with a tissue classifier that restricted analysis to pan- $\mathrm{CK}^{+}$areas within the predefined tumor area. The same algorithm was used for pre- and posttreatment biopsies. Cell counts were normalized to pan-CK tissue area (cells $\mu \mathrm{m}^{-2}$ ).

Primary antibodies were: CD8 (SP16; Lab Vision/Thermo Scientific), pancytokeratin (AE1/AE3 Alexa Fluor 488 conjugate; eBioscience), and CXCL12 (79018; R\&D Systems).

1. F. S. Hodi et al., Improved survival with ipilimumab in patients with metastatic melanoma. N. Engl. J. Med. 363, 711-723 (2010)

2. S. L. Topalian et al., Safety, activity, and immune correlates of anti-PD-1 antibody in cancer. N. Engl. J. Med. 366, 2443-2454 (2012)

3. J. R. Brahmer et al., Safety and activity of anti-PD-L1 antibody in patients with advanced cancer. N. Engl. J. Med. 366, 2455-2465 (2012).

4. P. Sharma, J. P. Allison, The future of immune checkpoint therapy. Science 348, 56-61 (2015).

5. T. N. Schumacher, W. Scheper, P. Kvistborg, Cancer neoantigens. Annu. Rev. Immunol. 37, 173-200 (2019).

6. L. M. McLane, M. S. Abdel-Hakeem, E. J. Wherry, CD8 T cell exhaustion during chronic viral infection and cancer. Annu. Rev. Immunol. 37, 457-495 (2019).

7. L. Zitvogel, Y. Ma, D. Raoult, G. Kroemer, T. F. Gajewski, The microbiome in cancer immunotherapy: Diagnostic tools and therapeutic strategies. Science 359, 1366-1370 (2018).

8. T. R. Flint et al., Tumor-induced IL-6 reprograms host metabolism to suppress antitumor immunity. Cell Metab. 24, 672-684 (2016).

9. E. Sahai et al., A framework for advancing our understanding of cancer-associated fibroblasts. Nat. Rev. Cancer 20, 174-186 (2020).

10. C. Germain et al., Presence of B cells in tertiary lymphoid structures is associated with a protective immunity in patients with lung cancer. Am. J. Respir. Crit. Care Med. 189, 832-844 (2014).

\section{Immunological Assays.}

Fluorescent in situ hybridization. RNAscope (Advanced Cell Diagnostics) was performed following the standard protocol. Frozen sections were fixed with $4 \%$ paraformaldehyde (PFA) for $15 \mathrm{~min}$ at $4{ }^{\circ} \mathrm{C}$ and dehydrated with sequential ethanol solution (50 to $100 \%$ ) at room temperature. Sections were incubated with pretreat IV for 30 min and washed in PBS before being hybridized with gene-specific probes (CCL19, FAP) for $2 \mathrm{~h}$ at $40{ }^{\circ} \mathrm{C}$ in a HybEZ oven. Sections were washed with wash buffer followed by incubations in sequential amplifers (Amp1-Amp4). Finally, sections were stained with DAPI and mounted with prolong antifade mountant. Images were taken by Leica SP8 confocal microscope and analyzed with ImageJ software.

Plasma Cell-Free DNA Analysis. Blood samples were collected into EDTAcontaining tubes and processed by a double-centrifugation protocol $(1,600 \times g$ for $10 \mathrm{~min} ; 14,000 \mathrm{rpm}$ for $10 \mathrm{~min})$ before storage at $-80^{\circ} \mathrm{C}$. Plasma DNA was extracted using QIAsymphony DSP Circulating DNA Kit (Qiagen) and DNA-sequencing libraries were prepared using the ThruPLEX Plasma-seq kit (Takara Bio). Unique DNA barcode sequences were introduced to allow pooled sequencing runs on HiSeq 4000 (Illumina) generating 150-bp long paired-end reads. Sequencing reads were subsequently aligned to the human reference genome (hg19) using BWA-mem. PCR and optical duplicates were marked using MarkDuplicates (Picard Tools) and excluded from downstream analysis along with reads of low mapping quality. Sequencing data in each sample were downsampled to 5 million reads to generate t-MAD scores (47) for estimating ctDNA levels inferred from a genome-wide copy number aberration profile. The segmentation step was summarized by a median value and a t-MAD threshold of 0.015 was used.

Cytokine Analysis. Serum was analyzed for CXCL8 using the MesoScale Discovery Human 10-plex Prolnflammatory Panel 1 kit (V-PLEX K15049D-2), as per the manufacturer's instructions. Analyses were conducted at the Core biochemical assay laboratory at Cambridge University Hospitals, NHS Foundation Trust.

Data Availability. All study data are included in the article and supporting information.

ACKNOWLEDGMENTS. We thank all patients; Purity Bundi, Breanna Demestichas, Nikos Demiris, Kate Donoghue, Alex Overhill, Richard Houghton, and Eva Serrao for help with data acquisition, trial management, and data illustration; and Hannah Meyer for critical appraisal of the manuscript. The study was carried out at/supported by the National Institute for Health Research (NIHR) Cambridge Clinical Research Facility. We acknowledge support from the Human Research Tissue Bank, supported by the NIHR Cambridge Biomedical Research Centre, for sample processing, and the Histopathology Core Facility at the Cancer Research UK Cancer Institute for sample immunostaining and imaging. Funding was provided by the SU2CLustgarten Foundation Dream Team and Cancer Research UK Institute core Grants C14303/A17197 and C9545/A29580. The Li Ka Shing Centre, where some of this research was performed, was generously funded by CK Hutchison Holdings Limited, the University of Cambridge, Cancer Research UK, The Atlantic Philanthropies, and a range of other donors. D.I.J. and F.M.R. were funded by Cancer Research UK C14303/A17197 and C9545/ A29580; T.J. was funded by Cancer Research UK C42738/A24868, National Institutes of Health 5P30CA045508-31, and the Pershing Square Innovation Fund. C.M.C. was funded by Experimental Medicine Initiative Clinical Lectureship.

11. F. Petitprez et al., B cells are associated with survival and immunotherapy response in sarcoma. Nature 577, 556-560 (2020).

12. B. A. Helmink et al., B cells and tertiary lymphoid structures promote immunotherapy response. Nature 577, 549-555 (2020)

13. R. Cabrita et al., Tertiary lymphoid structures improve immunotherapy and survival in melanoma. Nature 577, 561-565 (2020).

14. A. E. Denton, M. A. Linterman, Stromal networking: Cellular connections in the germinal centre. Curr. Opin. Immunol. 45, 103-111 (2017).

15. A. E. Denton, E. J. Carr, L. P. Magiera, A. J. B. Watts, D. T. Fearon, Embryonic FAP lymphoid tissue organizer cells generate the reticular network of adult lymph nodes. J. Exp. Med. 216, 2242-2252 (2019).

16. A. F. Olumi et al., Carcinoma-associated fibroblasts direct tumor progression of initiated human prostatic epithelium. Cancer Res. 59, 5002-5011 (1999).

17. P. Garin-Chesa, L. J. Old, W. J. Rettig, Cell surface glycoprotein of reactive stromal fibroblasts as a potential antibody target in human epithelial cancers. Proc. Natl. Acad. Sci. U.S.A. 87, 7235-7239 (1990).

18. M. Kraman et al., Suppression of antitumor immunity by stromal cells expressing fibroblast activation protein-alpha. Science 330, 827-830 (2010).

19. A. Orimo et al., Stromal fibroblasts present in invasive human breast carcinomas promote tumor growth and angiogenesis through elevated SDF-1/CXCL12 secretion. Cell 121, 335-348 (2005). 
20. C. Feig et al., Targeting CXCL12 from FAP-expressing carcinoma-associated fibroblasts synergizes with anti-PD-L1 immunotherapy in pancreatic cancer. Proc. Natl. Acad. Sci. U.S.A. 110, 20212-20217 (2013).

21. W. B. Zhang et al., A point mutation that confers constitutive activity to CXCR4 reveals that T140 is an inverse agonist and that AMD3100 and ALX40-4C are weak partial agonists. J. Biol. Chem. 277, 24515-24521 (2002).

22. T. J. Schall, A. E. I. Proudfoot, Overcoming hurdles in developing successful drugs targeting chemokine receptors. Nat. Rev. Immunol. 11, 355-363 (2011).

23. C. W. Hendrix et al.; AMD3100 HIV Study Group, Safety, pharmacokinetics, and antiviral activity of AMD3100, a selective CXCR4 receptor inhibitor, in HIV-1 infection. J. Acquir. Immune Defic. Syndr. 37, 1253-1262 (2004).

24. A. Peled et al., Dependence of human stem cell engraftment and repopulation of NOD/SCID mice on CXCR4. Science 283, 845-848 (1999)

25. A. J. Gentles et al., The prognostic landscape of genes and infiltrating immune cells across human cancers. Nat. Med. 21, 938-945 (2015).

26. S. Siegert, S. A. Luther, Positive and negative regulation of $\mathrm{T}$ cell responses by fibroblastic reticular cells within paracortical regions of lymph nodes. Front. Immunol. 3, 285 (2012).

27. A. E. Denton, E. W. Roberts, M. A. Linterman, D. T. Fearon, Fibroblastic reticular cells of the lymph node are required for retention of resting but not activated CD8+ T cells. Proc. Natl. Acad. Sci. U.S.A. 111, 12139-12144 (2014).

28. J. Reeve et al., Molecular diagnosis of $\mathrm{T}$ cell-mediated rejection in human kidney transplant biopsies. Am. J. Transplant. 13, 645-655 (2013).

29. P. F. Halloran et al., Potential impact of microarray diagnosis of T cell-mediated re jection in kidney transplants: The INTERCOM study. Am. J. Transplant. 13, 2352-2363 (2013).

30. E. A. Kautto et al., Performance evaluation for rapid detection of pan-cancer microsatellite instability with MANTIS. Oncotarget 8, 7452-7463 (2017).

31. M. Goldman et al., The UCSC Xena platform for cancer genomics data visualization and interpretation. bioRxiv:10.1101/326470 (28 August 2018).

32. N. Riaz et al., Tumor and microenvironment evolution during immunotherapy with nivolumab. Cell 171, 934-949.e16 (2017).

33. T. N. Gide et al., Distinct immune cell populations define response to anti-PD-1 monotherapy and anti-PD-1/anti-CTLA-4 combined therapy. Cancer Cell 35, 238-255.e6 (2019).
34. J. Griss et al., B cells sustain inflammation and predict response to immune checkpoint blockade in human melanoma. Nat. Commun. 10, 4186 (2019).

35. J. C. M. Wan et al., Liquid biopsies come of age: Towards implementation of circulating tumour DNA. Nat. Rev. Cancer 17, 223-238 (2017).

36. L. Cabel et al., Clinical potential of circulating tumour DNA in patients receiving anticancer immunotherapy. Nat. Rev. Clin. Oncol. 15, 639-650 (2018).

37. V. Anagnostou et al., Dynamics of tumor and immune responses during immune checkpoint blockade in non-small cell lung cancer. Cancer Res. 79, 1214-1225 (2019).

38. C. A. Parkinson et al., Exploratory analysis of TP53 mutations in circulating tumour DNA as biomarkers of treatment response for patients with relapsed high-grade serous ovarian carcinoma: A retrospective study. PLoS Med. 13, e1002198 (2016).

39. M. F. Sanmamed et al., Serum interleukin-8 reflects tumor burden and treatment response across malignancies of multiple tissue origins. Clin. Cancer Res. 20, 5697-5707 (2014).

40. M. F. Sanmamed et al., Changes in serum interleukin-8 (IL-8) levels reflect and predict response to anti-PD-1 treatment in melanoma and non-small-cell lung cancer patients. Ann. Oncol. 28, 1988-1995 (2017).

41. K. C. Yuen et al., High systemic and tumor-associated IL-8 correlates with reduced clinical benefit of PD-L1 blockade. Nat. Med. 26, 693-698 (2020).

42. F. S. Hodi et al., Evaluation of immune-related response criteria and RECIST v1.1 in patients with advanced melanoma treated with pembrolizumab. J. Clin. Oncol. 34, 1510-1517 (2016).

43. J. D. Wolchok et al., Guidelines for the evaluation of immune therapy activity in solid tumors: Immune-related response criteria. Clin. Cancer Res. 15, 7412-7420 (2009)

44. B. Bockorny et al., BL-8040, a CXCR4 antagonist, in combination with pembrolizumab and chemotherapy for pancreatic cancer: The COMBAT trial. Nat. Med. 26, 878-885 (2020).

45. S. Nayar et al., Immunofibroblasts are pivotal drivers of tertiary lymphoid structure formation and local pathology. Proc. Natl. Acad. Sci. U.S.A. 116, 13490-13497 (2019).

46. M. I. Love, W. Huber, S. Anders, Moderated estimation of fold change and dispersion for RNA-seq data with DESeq2. Genome Biol. 15, 550 (2014).

47. F. Mouliere et al., Enhanced detection of circulating tumor DNA by fragment size analysis. Sci Transl. Med. 10, eaat4921 (2018). 